\title{
An Optimization Model for Advanced Biofuel Production Based on Bio-oil Gasification
}

\author{
Qi Li and Guiping $\mathrm{Hu}^{*}$ \\ Industrial and Manufacturing Systems Engineering, Iowa State University, \\ Ames, IA, 50011, United States \\ gphuaiastate.edu
}

\begin{abstract}
Biomass can be converted to transportation fuels through gasification. However, commercialization of biomass gasification has been hampered by its high capital and operating costs, in addition to the difficulties of transporting bulky solid biomass over a long distance. A novel approach is to convert biomass to bio-oil at widely distributed small-scale fast pyrolysis plants, transport the bio-oil to a centralized location, gasify the bio-oil to syngas, and upgrade the syngas to transportation fuels. In this paper, a two-stage stochastic programming is formulated. The first-stage makes the capital investment decisions including the locations and capacities of the bio-facilities (fast pyrolysis and refining facility) while the second-stage determines the biomass and biofuels flows. This paper aims to find the optimal design of the supply chain for this certain path considering uncertainties in biomass yield, biofuel price and transportation costs.
\end{abstract}

Keywords: stochastic programming, bio-oil gasification, supply chain.

\section{$1 \quad$ Introduction}

Second generation biofuel is a potential substitute for petroleum-based fuel in the perspectives of environmental, economic, and social benefits. [1]. According to the revised Renewable Fuel Standard (RFS2), at least 36 billion gallons per year of renewable fuels will be produced by 2022, of which at least 16 billion gallons per year will be from cellulosic biofuels [2]. However, the cellulosic biofuel volume standard for 2012 is only 10.45 million gallons per year according U.S. Environmental Protection Agency (EPA) in 2011 [3].

Biomass can be converted to transportation fuels by various methods. Fast pyrolysis and gasification are two of the most prominent technologies for thermochemical conversion of biomass. Fast pyrolysis produces bio-oil, bio-char and non-condensable gases [4]. However, commercialization of the biomass gasification has been hampered by its high capital and operating costs due to the challenge of transporting bulky solid biomass over a long distance, processing the solid feedstock at high pressure, and removing contaminants from gas stream. Feedstock production and logistics constitute $35 \%$ or more of the total production costs of advanced biofuel [5]. And

\footnotetext{
* Corresponding author.
} 
logistics associated with moving biomass from land to bio-refinery can make up 50$75 \%$ of the feedstock costs [6]. A novel approach for reducing these costs is to convert biomass to bio-oil at distributed small-scale fast pyrolysis processing plants, transport the bio-oil to a centralized location, gasify the bio-oil to syngas, and upgrade the syngas to transportation fuels. There is a rich literature on supply chain network design. Shah [7] reviewed the precious studies in modeling, planning, and scheduling with some real world examples. An et al. [8] compared the supply chain research on petroleum based fuel and biofuel. However, only a few literature concerns the uncertainty. Kim et al. [9] considered a two-stage stochastic model to determine the capacities and location of the bio-refineries. Marvin et al. [10] gave a mixed integer linear programming to determine optimal locations and capacities of bio-refineries. As a result, it is of importance to build an optimization model to deal with the uncertainties such as biomass supply, shipping cost and biofuel demands.

The rest of the paper is organized as follows: in Section 2, the problem statement and assumptions is presented. Then, we discuss the deterministic linear programming model and the two-stage stochastic programming models for this problem in Section 3. Section 4 gives a brief discussion of settings and result of a toy case study. Finally, we conclude the paper in Section 5 with summary and future research directions.

\section{Problem Statement and Assumptions}

The biofuel industry is highly affected by the uncertainties among the supply chain such as biomass supply, shipping cost and biofuel demands. Stochastic programming is one of the most widely used methods to consider the impacts of decision making under uncertainties. This paper aims to provide a mathematical programming framework with a two-stage stochastic programming approach to deal with the uncertainties among the biofuel industry. The optimality model provides suggestions about the capital investment decisions and logistic decisions of this pathway.

In this model, several assumptions are made. Biomass supply is assumed from the county level. Candidate fast pyrolysis and the refining facilities are assumed at the county centroid. Only one centralized refining facility is being planned and the decentralized fast pyrolysis facilities can be of several capacity levels (low, medium and high levels). It is assumed that the unit collection cost of biomass includes the feedstock inventory cost.

\section{Model Formulation}

In this section, we introduce both the deterministic and stochastic model for this biofuel supply chain design problem. The deterministic mixed integer linear programming model is first introduced as a baseline model and then a two-stage stochastic model is discussed to address the uncertainties in the supply chain design problem.

\subsection{Deterministic Mixed Integer Linear Programming Model}

In the deterministic mixed integer linear programming model, all the data and modeling parameters are assumed to known with certainty. The notations used in this model are listed in Table 1. 
Table 1. Notations for Deterministic Linear Programming Model

\begin{tabular}{|c|c|c|}
\hline \multicolumn{3}{|c|}{ Subscripts } \\
\hline$i$ & $1,2, \ldots, I$ & Biomass supply locations \\
\hline j & $1,2, \ldots, J$ & Candidate fast pyrolysis facility locations \\
\hline$k$ & $1,2, \ldots, K$ & Gasoline and diesel fuel demand locations \\
\hline$l$ & $1,2, \ldots, L$ & Allowed fast pyrolysis capacity levels \\
\hline$m$ & $1,2, \ldots, M$ & Candidate refining facility locations \\
\hline \multicolumn{3}{|c|}{ Decision Variables } \\
\hline$x_{i j}$ & \multicolumn{2}{|c|}{$\begin{array}{l}\text { Amount of biomass transported from supply location } i \text { to candidate fast } \\
\text { pyrolysis facility location } j\end{array}$} \\
\hline$y_{j m}$ & \multicolumn{2}{|c|}{$\begin{array}{l}\text { Amount of bio-oil transported from candidate fast pyrolysis facility loca- } \\
\text { tion } j \text { to candidate refining facility location } m\end{array}$} \\
\hline$z_{m k}$ & \multicolumn{2}{|c|}{$\begin{array}{l}\text { Amount of gasoline and diesel fuels transported from refining facility loca- } \\
\text { tion } m \text { to demand location } k\end{array}$} \\
\hline$a_{j l}$ & \multicolumn{2}{|c|}{$\begin{array}{l}\text { Whether a fast pyrolysis facility of capacity level } l \text { is planned at the candi- } \\
\text { date facility location } j \text { (binary variable) }\end{array}$} \\
\hline$g_{m}$ & \multicolumn{2}{|c|}{$\begin{array}{l}\text { Whether a refining facility exists in candidate refining facility location } m \\
\text { (binary variable) }\end{array}$} \\
\hline \multicolumn{3}{|c|}{ Parameters } \\
\hline$B$ & \multicolumn{2}{|c|}{ Total budget } \\
\hline $\bar{C}^{U P}$ & \multicolumn{2}{|c|}{ Capital cost of the centralized refining facility } \\
\hline$C_{l}^{\text {Cap }}$ & \multicolumn{2}{|c|}{ Capital cost of the decentralized fast pyrolysis facility at level $l$} \\
\hline$P_{k}$ & \multicolumn{2}{|c|}{ Gasoline and diesel fuels price at demand location $k$} \\
\hline$D_{k}$ & \multicolumn{2}{|c|}{ Gasoline and diesel fuels demand at demand location $k$} \\
\hline$P e_{k}$ & \multicolumn{2}{|c|}{ Penalty for not meeting the demand at demand location $k$} \\
\hline$P e_{k}{ }^{\prime}$ & \multicolumn{2}{|c|}{ Penalty for exceeding the demand at demand location $k$} \\
\hline$\frac{C_{k}}{C_{i}^{C o l}}$ & \multicolumn{2}{|c|}{ Unit biomass collecting cost at supply location $i$} \\
\hline$\frac{C_{i}}{C^{M O}}$ & \multicolumn{2}{|c|}{ Unit conversion cost from dry biomass to bio-oil } \\
\hline$C^{O F}$ & \multicolumn{2}{|c|}{ Unit conversion cost from bio-oil to biofuels } \\
\hline$C_{i j}^{B M}$ & \multicolumn{2}{|c|}{$\begin{array}{l}\text { Unit biomass shipping cost from supply location } i \text { to candidate fast pyroly- } \\
\text { sis facility location } j\end{array}$} \\
\hline$C_{j m}^{B O}$ & \multicolumn{2}{|c|}{$\begin{array}{l}\text { Unit bio-oil shipping cost from candidate fast pyrolysis facility location } \\
j \text { to candidate refining facility location } m\end{array}$} \\
\hline$C_{m k}^{B F}$ & \multicolumn{2}{|c|}{$\begin{array}{l}\text { Unit biofuel shipping cost from candidate refining facility location } m \text { to } \\
\text { demand location } k\end{array}$} \\
\hline$\overline{U_{l}}$ & \multicolumn{2}{|c|}{ Capacity of fast pyrolysis facility at level $l$} \\
\hline $\begin{array}{l}l \\
V\end{array}$ & \multicolumn{2}{|c|}{ Capacity of refining facility } \\
\hline$S_{i}$ & \multicolumn{2}{|c|}{ Available biomass feedstock at location $i$} \\
\hline$\alpha$ & \multicolumn{2}{|c|}{ Sustainability factor } \\
\hline$\beta$ & \multicolumn{2}{|c|}{ Conversion factor from wet biomass to dry biomass } \\
\hline$\gamma$ & \multicolumn{2}{|c|}{ The loss factor of biomass during collection and transportation } \\
\hline & \multicolumn{2}{|c|}{ Conversion ratio, ton of bio-oil per ton of dry biomass } \\
\hline$\theta_{2}$ & \multicolumn{2}{|c|}{ Conversion ratio, ton of gasoline per ton of bio-oil } \\
\hline
\end{tabular}




\subsubsection{Objective Function}

The objective of this model is to maximize the total profit, which can be defined as the income from selling the biofuel subtracted by the penalty and the total cost. There is a penalty on not meeting the demand and an additional storage penalty for any surplus production. Different types of costs are considered in this model including the capital investment, collection cost, conversion cost, and shipping cost [11]. The shipping cost includes the transportation costs for biomass feedstock, intermediate bio-oil, and upgrading transportation fuels.

\subsubsection{Constraints in the Model}

The following constraint is used to ensure that the total capital cost does not exceed the budget.

$$
B-C^{U P}-\sum_{j=1}^{J} \sum_{l=1}^{L} C_{l}^{C a p} a_{j l} \geq 0
$$

The total amount of biomass transported from supply location $i$ to candidate fast pyrolysis facility locations should not exceed the available feedstock at each supply location where $\alpha$ is the sustainability factor.

$$
\sum_{j=1}^{J} x_{i j} \leq(1-\alpha) S_{i}, \forall i
$$

The capacity constraints are used in the model. The loss factor $\gamma \in[0,1)$ is the fraction weight loss of biomass during the collection and transportation and $\beta$ is the conversion factor from wet biomass to dry biomass.

$$
\begin{gathered}
\sum_{l=1}^{L} U_{l} a_{j l}-(1-\gamma) \beta \sum_{i=1}^{I} x_{i j} \geq 0, \forall j \\
V g_{m}-\sum_{j=1}^{J} y_{j m} \geq 0, \forall m
\end{gathered}
$$

There should be no more than one fast pyrolysis facility exists in each candidate facility location. And only one centralized refining facility is built.

$$
\begin{gathered}
\sum_{l=1}^{L} a_{j l} \leq 1, \forall j \\
\sum_{m=1}^{M} g_{m}=1
\end{gathered}
$$

We assume that biomass is converted to bio-oil with a conversion ratio $\theta_{1}$ and bio-oil is converted to biofuel with a conversion ratio $\theta_{2}$. Thus, we have the following conversion balance constraints:

$$
\begin{aligned}
& (1-\gamma) \beta \theta_{1} \sum_{i=1}^{I} x_{i j}-\sum_{m=1}^{M} y_{j m}=0, \forall j \\
& \theta_{2} \sum_{j=1}^{J} \sum_{m=1}^{M} y_{j m}-\sum_{m=1}^{M} \sum_{k=1}^{K} z_{m k}=0
\end{aligned}
$$




\subsubsection{Summary of the Deterministic Model}

The deterministic linear programming model is formulated as follows:

$\max \zeta=$ income - penalty - cost $=\sum_{k=1}^{K}\left(P_{k} \sum_{m=1}^{M} z_{m k}\right)-\left\{\left(D_{k}-\sum_{m=1}^{M} z_{m k}\right)_{+} *\right.$ $\left.P e_{k}+\left(\sum_{m=1}^{M} z_{m k}-D_{k}\right)_{+} * P e_{k}^{\prime}\right\}-\left\{\sum_{j=1}^{J} \sum_{l=1}^{L} C_{l}^{C a p} a_{j l}+\sum_{i=1}^{I} \sum_{j=1}^{J} C_{i}^{C o l} x_{i j}+\right.$ $C^{M O}(1-\gamma) \beta \sum_{i=1}^{I} x_{i j}+C^{O F} \sum_{j=1}^{J} \sum_{m=1}^{M} y_{j m}+\sum_{i=1}^{I} \sum_{j=1}^{J} C_{i j}^{B M} x_{i j}+$ $\sum_{j=1}^{J} \sum_{m=1}^{M} C_{j m}^{B O} y_{j m}+\sum_{m=1}^{M} \sum_{k=1}^{K} C_{m k}^{B F} z_{m k}$

s.t. Constraints (1) - (8), $x_{i j}, y_{j m}, z_{m k} \geq 0, a_{j l}, g_{m} \in\{0,1\}, \forall i, j, l, m, k$

This mixed integer linear programming model maximizes the total profit by giving capital investment and logistics decisions at the same time in deterministic case. It's the baseline model for next step.

\subsection{Two-Stage Stochastic Programming Model}

In this paper, we consider the uncertainties of the shipping cost, available biomass feedstock and gasoline and diesel fuels prices. The stochastic parameters in this study are assumed to be discretely distributed. We use subscript $s$ to represent scenario with probability $P r_{s}$ and add this subscript to some decision variables and parameters. $\lambda_{s}$ is percentage change of the shipping cost in scenario $s$ comparing to the base scenario. The two-stage stochastic programming model is formulated as follows:

$$
\begin{aligned}
& \max \zeta=-\sum_{j=1}^{J} \sum_{l=1}^{L} C_{l}^{C a p} a_{j l}+\sum_{s=1}^{S} P r_{s}\left\{\sum_{\mathrm{k}=1}^{K} \sum_{m=1}^{M}\left(P_{k s} z_{m k s}\right)-\left(\left(D_{k}-\right.\right.\right. \\
& \left.\left.\sum_{m=1}^{M} z_{m k s}\right)_{+} * P e_{k}+\left(\sum_{m=1}^{M} z_{m k s}-D_{k}\right)_{+} * P e_{k}^{\prime}\right)-\left(\sum_{i=1}^{I} \sum_{j=1}^{J} C_{i}^{C o l} x_{i j s}+\right. \\
& C^{M O}(1-\gamma) \beta \sum_{i=1}^{I} x_{i j s}+C^{O F} \sum_{j=1}^{J} \sum_{m=1}^{M} y_{j m s}+\left(\lambda _ { s } \left(\sum_{i=1}^{I} \sum_{j=1}^{J} C_{i j}^{B M} x_{i j s}+\right.\right. \\
& \left.\left.\left.\left.\sum_{j=1}^{J} \sum_{m=1}^{M} C_{j m}^{B O} y_{j m s} \sum_{m=1}^{M} \sum_{k=1}^{K} C_{m k}^{B F} z_{m k s}\right)\right)\right)\right\}
\end{aligned}
$$

s.t. Constraints (1), (5), (6).

$$
\begin{gathered}
\sum_{j=1}^{J} x_{i j s} \leq(1-\alpha) S_{i s}, \forall i, \forall s \\
\sum_{l=1}^{L} U_{l} a_{j l}-(1-\gamma) \beta \sum_{i=1}^{I} x_{i j s} \geq 0, \forall j, \forall s \\
V g_{m}-\sum_{j=1}^{J} y_{j m s} \geq 0, \forall m, \forall s \\
(1-\gamma) \beta \theta_{1} \sum_{i=1}^{I} x_{i j s}-\sum_{m=1}^{M} y_{j m s}=0, \forall j, \forall s \\
\theta_{2} \sum_{j=1}^{J} \sum_{m=1}^{M} y_{j m s}-\sum_{m=1}^{M} z_{m k s}=0, \forall s \\
x_{i j s}, y_{j m s}, z_{m k s} \geq 0, a_{j l}, g_{m} \in\{0,1\}, \forall i, j, k, m, l, s
\end{gathered}
$$

In this model, the first-stage decision variables are $a_{j l}$ and $g_{m}$, which make the capital investment decisions including the locations and capacities for the conversion facilities. While the second-stage decision variables $x_{i j s}, y_{j m s}$, and $z_{m k s}$ determine the biomass and biofuels flows. Constraints (1), (5), and (6) are the first-stage 
constraints, these constraints remain the same. The rest of the constraints will change accordingly to the set of scenarios.

\section{$4 \quad$ Case Study}

A case study based on state of Iowa has been carried out to illustrate the modeling framework. Historical data from EIA, USDA, and Census Bureau supplemented by the literatures have been employed in the case study. Three levels of uncertainties for each parameter have been considered. The 27 scenarios are assumed to have equal probability. The results show that in the deterministic case, we should build 51 distributed fast pyrolysis plants (one low-capacity facility, no medium-capacity facility and 50 high-capacity facilities). The yearly profit is about 886 million dollars. The capital investment could be recovered in 6 years with the interest rate of $10 \%$. While in the stochastic case, 63 distributed fast pyrolysis plants (one low-capacity facility, 14 medium-capacity facilities and 48 high-capacity facilities). It is observed that the stochastic programming modeling framework demonstrates superior economic outcome than the deterministic case and the value of the stochastic solution (VSS) is about 65 million dollars per year.

\section{Conclusion}

This paper provides a mathematical programming framework with a two-stage stochastic programming approach to deal with the uncertainties in the supply chain design among the biofuel industry. The optimality model provides suggestions for the decision makers on the capital investment decisions and logistic decisions of the thermochemical conversion pathway based on bio-oil gasification. A case study has been carried out to illustrate and validate the modeling framework. This paper provides a preliminary framework and initiates a future research direction for uncertainty analysis in biofuel supply chain design. Additional constraints on the detailed process design can be incorporated to the modeling framework. It should also be noted that additional uncertainties can be considered and more realistic criterion to generate scenarios for the stochastic programming can be investigated.

\section{References}

1. Carriquiry, M.A., Du, X., Timilsina, G.R.: Second generation biofuels: Economics and policies. Energy Policy 39(7), 4222-4234 (2011)

2. Schnepf, R.: Renewable fuel standard (RFS): overview and issues. Diane Publishing (2011)

3. EPA, EPA finalizes 2012 renewable fuel standards (2011)

4. Brown, R.C.: Biorenewable resources. Iowa State Press (2003)

5. Wooley, R., Ruth, M., Sheehan, J., Ibsen, K., Majdeski, H., Galvez, A.: Lignocellulosic biomass to ethanol process design and economics utilizing co-current dilute acid prehydrolysis and enzymatic hydrolysis current and futuristic scenarios (No. NREL/TP580-26157). National Renewable Energy Lab Golden Co. (1999) 
6. Grant, D., Hess, J.R., Kenney, K., Laney, P., Muth, D., Pryfogle, P., Radtke, C., Wright, C.: Feasibility of a producer-owned ground-straw feedstock supply system for bioethanol and other products, p. 115. INL, Idaho (2006)

7. Shah, N.: Process industry supply chains: Advances and challenges. Computers \& Chemical Engineering 29(6), 1225-1236 (2005)

8. An, H., Wilhelm, W.E., Searcy, S.W.: Biofuel and petroleum-based fuel supply chain research: a literature review. Biomass and Bioenergy 35(9), 3763-3774 (2011)

9. Kim, J., Realff, M.J., Lee, J.H.: Optimal design and global sensitivity analysis of biomass supply chain networks for biofuels under uncertainty. Computers \& Chemical Engineering 35(9), 1738-1751 (2011)

10. Alex Marvin, W., Schmidt, L.D., Benjaafar, S., Tiffany, D.G., Daoutidis, P.: Economic optimization of a lignocellulosic biomass-to-ethanol supply chain. Chemical Engineering Science 67(1), 68-79 (2012)

11. Li, Y., Brown, T., Hu, G.: An optimization model to determine the capacities and locations for a thermochemical biorefinery supply network. Iowa State University. Technical report 